\title{
Analysis of Workplaces Supply in a Production Company
}

\author{
Vieroslav Molnár ${ }^{1}$, Petr Průša ${ }^{2, *}$, Jan Strohmandl ${ }^{3}$, and Michal Legát ${ }^{1}$ \\ ${ }^{1}$ Technical University of Košice, BERG Faculty, Letná 9, 04200 Košice, Slovakia \\ ${ }^{2}$ University of Pardubice, Department of Transport Management, Marketing and Logistics, \\ Studentská 95, 53210 Pardubice, Czech Republic \\ ${ }^{3}$ Tomas Bata University in Zlín, nám. T. G. Masaryka 5555, 76001 Zlín, Czech Republic
}

\begin{abstract}
The paper deals with the analysis of the supply of workplaces and the description of the related activities. Workplaces are supplied using specific supply means. The individual supplies used in the supply system, a description of the main activities and the principle of work are characterized. There are five alternative proposals for improving the supply of production workplaces. A proportional index method was used to select the optimal variant of the supply solution for the production workplaces. The optimal solution proposal is to add the transport lanes in the production hall.
\end{abstract}

\section{Introduction}

In today's modern times, the workplaces supplying is an important part of the especially production companies, that want to succeed in the global market [1]. At present, different supply systems are used in the world, and one of them is the system of supplying workplaces with supply means [2]. The successful functioning of the company in a competitive struggle cannot be realized without functional supply logistics [3, 4]. Supplying workplaces is a complicated process both in terms of technical difficulty and security. Supplying ensures the transfer of material from the warehouse to the place of production and moving the finished products from the place of production to the place of storage [5]. Transferring material or goods in this space has a certain distance that takes some time [6]. Every manufacturing company is trying to shorten this time by appropriate measures [7]. The company also strives to find optimal solutions that do not have a big economic impact on the business [8]. Such solutions can be determined by using various methods that evaluate different options on the basis of the given criteria [9].

By analysing the company's supply system, it is possible to identify deficiencies that cannot be detected without defining its system elements and elements that operate on the system $[10,11]$.

* Corresponding author: Petr.Prusa@upce.cz 


\section{The current state of workplaces supply}

If a company wants to maintain its competitiveness, it must take prompt responses to the constantly changing market conditions. That is the reason the modern logistics solutions and external procurement are used [12, 13], which are mainly focused on company costs saving. In a surveyed enterprise, production planning is realized in a week ahead interval and for this reason, a supply scheme is created managed by a production program [14]. This is related to the utilization of individual supply stations and also to all supply trains $\mathrm{T} 1 \div \mathrm{T} 5$ that transport material to production lines at times recorded by the enterprise information system.

\subsection{Train T1}

Train T1 consists of a tractor and three gantry wagons that are used to transport the full transport boxes from the production hall to the warehouse and transport empty boxes from the warehouse to the production hall. Wagons are characterized by their simple handling, high stability and the control of all wheels as required. An advantage is the loading of transport boxes from both sides of the wagon. In the wagons, a wheeled trolley of the exact dimensions is used, which fits on the gantry wagon included the loaded transport box (Fig. 1). Gantry wagons are also provided with transport position indicators.
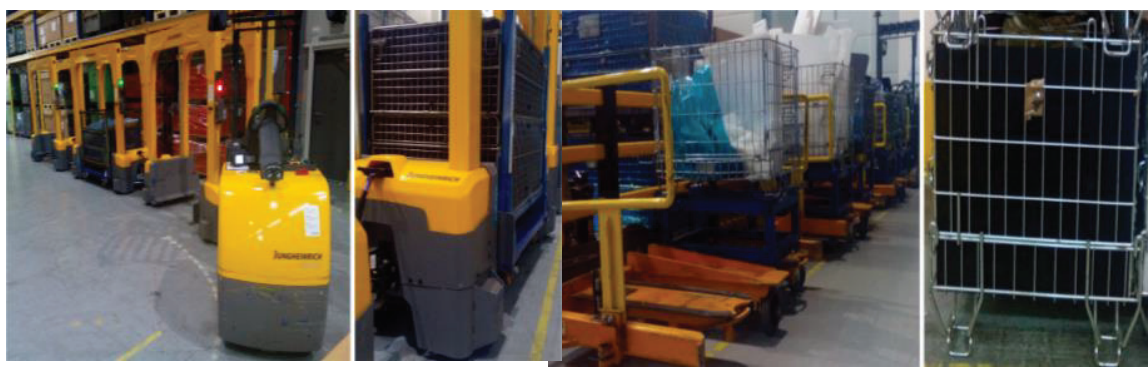

Fig. 1. Train T1 with the gantry wagons left and train T2 with transport basket. Source: authors

\subsection{Train T2}

Train T2 supplies the production workplaces with a medium size material of higher weight (Fig. 1). The material is stored in transport baskets. Baskets with the material are picked by a stacker located in the aisle between shelf racks with stored baskets. The stacker places them on transport wheeled trolley adapted for the wagons of this train. The transport capacity of the wagons is limited to 6 transport baskets. Wagons are flexible. Their weak point is the unloading of the transport baskets only from the right side of the wagons.

\subsection{Train T3}

Train 3 supplies production workplaces with materials of smaller dimensions and lower weight in crates (Fig. 2). There are used four types of crates in the company, which have different dimensions and volumes for different small materials and are stackable with each other (Fig. 2). The train is equipped with a specific wagon intended only for the transport and supply of workplaces by transport crates and a smaller wagon adapted for a metal cage to collect empty transport crates that are collected at the production workplaces and transported back to the warehouse. 

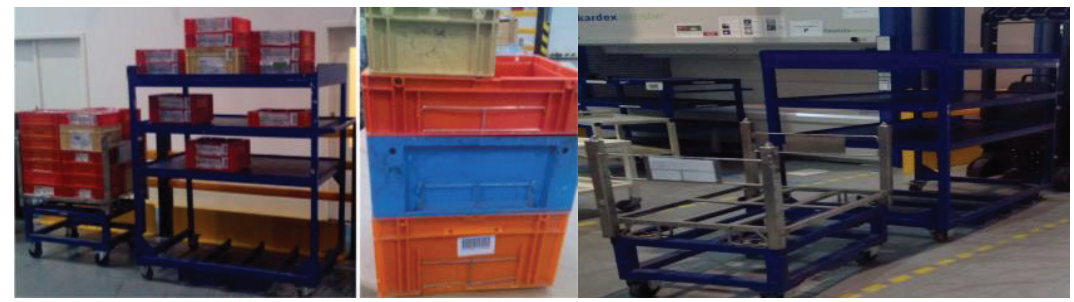

Fig. 2. Train T3 and the crates left and train T4 right. Source: authors

\subsection{Train T4}

Train 4 (Fig. 2) is used to supply manufacturing workplaces with small specific materials stored in the maximum of 15 crates. The material is picked by entering its position on the control panel using four modern cabinet warehouses according to the production requirements.

\subsection{Train T5}

The purpose of the Train T5 (Fig. 3) is to supply workplaces exclusively in the case of a priority production requirement for a particular type and quantity of material stored in transport baskets and crates (stored in metal cages). This train does not enter the production hall except for priority requirements.

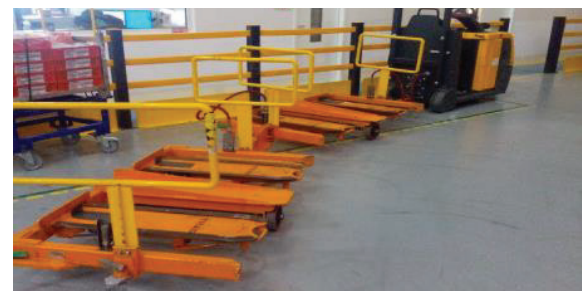

Fig. 3. Train T5. Source: authors

\subsection{Loading and unloading of trains}

The design of the production hall and the points of loading and unloading by Train T1-T4 are shown in Fig. 4.
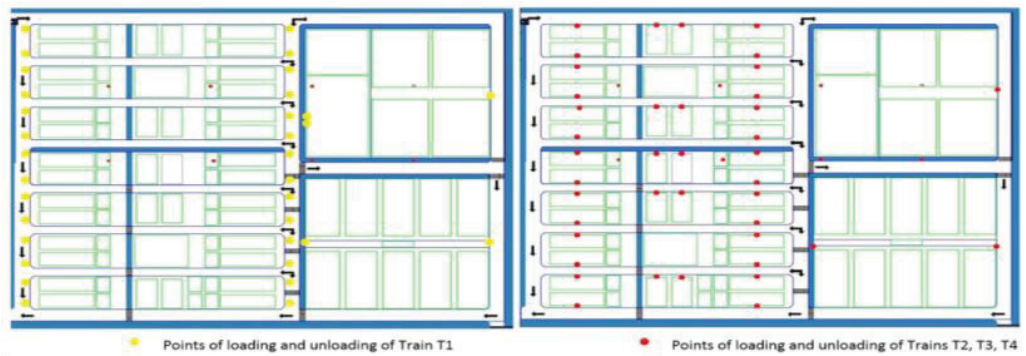

Fig. 4. The design of the production hall and the points of loading and unloading by Train T1-T4.1. Source: authors 


\section{Alternatives to improving workplaces supply}

The most important factor in the proposed alternatives for improving the supply of workplaces in the selected manufacturing enterprise is their economic efficiency. The cheap and the most immediate improvement in supply efficiency must count on the fact that production takes place continuously during all business days. In the next section, five possible alternatives to improving the supply of workplaces will be presented.

\subsection{Adding transport lanes in the production hall}

Transport routes in the production hall contain a single lane allowing only one supply mean to be driven without the possibility of being overtaken by the other means due to safety at the workplace. When overtaking in one narrow lane, there is a high probability of possible collisions with other supplying means of workplaces, production lines and, last but not least, with employees. For these reasons, traffic jams arise in the production hall (Fig. 5).

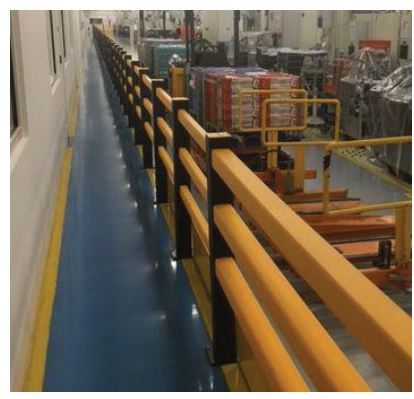

Fig. 5. Traffic jam in the production hall. Source: authors

If such a case occurs in the production hall, all the trains standing in the row must wait until the first in the column moves to the next position where the same situation may arise if the train standing behind goes in the same direction. Therefore, by solving this relatively frequent supply efficiency situation, it is precisely the new transport routes creation or extension them of two lanes (Fig. 6) and, at some places in the production hall, the introduction of bi-directional lanes with the possibility of overtaking.

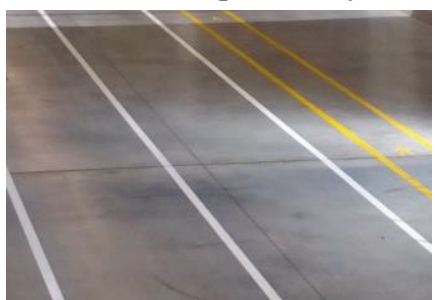

Fig. 6. A demonstration of a two-lane transport route. Source: authors

\subsection{Reducing the number of unloading places at production lines}

The essence of the proposal to reduce the number of unloading places at production lines is to minimize the movement of a worker with the supplying means. Any unnecessary movement of the worker additionally increases the supply time of workplaces. In this case, one production line would only be supplied in one place or the supply means would concentrate the material according to the specified production requirements at a precisely defined location in close proximity to the production line from where it would be available for the whole production line (Fig. 7). 


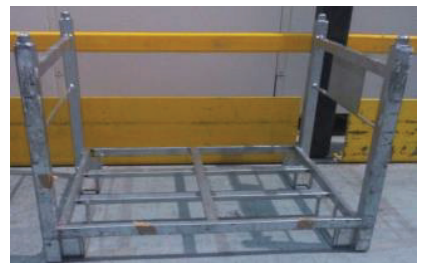

Fig. 7. Reserved place for material unloading. Source: authors

\subsection{Supply means differed by priority}

The proposal of driving supplying means according to certain priority criteria is to drive visually labeled supplying means, which have a preference in movement along the transport routes in the production hall. The main purpose of this proposal is to prevent the situation from occurring when an urgent demand on the production workplaces is to be met and this prompt fulfillment is not possible because of an obstacle in the form of any supplying means that, for example, standing in the lane (Fig. 8). The solution is to prioritize the supply means to transport at intersections and at certain points of transport routes in the production hall.

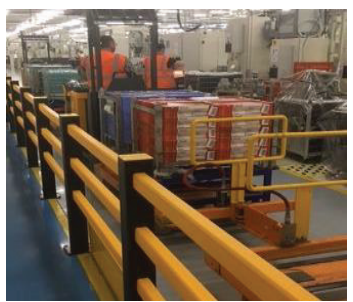

Fig. 8. Blocking of priority production requirement. Source: authors

\subsection{Hybrid supply means}

Hybrid supply means are formed by the creation of four supply means, which are a combination of four trains $\mathrm{T} 1 \div \mathrm{T} 4$. They would be able to meet all production requirements. In their optimal deployment, they would be able to flexibly supply highperformance production lines.

\subsection{Automated supply means}

Automated supply means are reliable in supplying production lines and able to flexibly change their transport routes as required. They are guided by magnetic strips glued on the floor throughout the manufacturing plant. The equipment includes automatic unloading and loading of material as well as a security scanner, which indicates movement in its surroundings (Fig. 9).

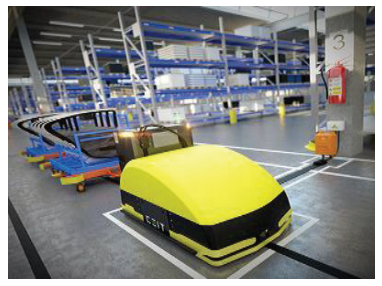

Fig. 9. Automated supply means. Source: [15] 


\section{Results and discussion}

Authors and several experts participated on criteria defining and evaluating and classification individual proposals. Table 1 shows which criteria have each suggested alternative. Its basic evaluating of alternatives.

Table 1. The strengths and weaknesses of proposed solutions. Source: authors

\begin{tabular}{|l|c|c|c|c|c|}
\hline \multirow{2}{*}{\multicolumn{1}{|c|}{ Criteria }} & \multicolumn{5}{|c|}{ Suggested alternatives } \\
\cline { 2 - 6 } & $\mathbf{1}$ & $\mathbf{2}$ & $\mathbf{3}$ & $\mathbf{4}$ & $\mathbf{5}$ \\
\hline Flexibility & $\mathbf{X}$ & $\mathbf{X}$ & $\mathbf{X}$ & $\mathbf{X}$ & $\mathbf{X}$ \\
\hline Quick installation & & $\mathbf{X}$ & $\mathbf{X}$ & & \\
\hline Economic efficiency & $\mathbf{X}$ & $\mathbf{X}$ & $\mathbf{X}$ & & \\
\hline Capacity utilization & $\mathbf{X}$ & $\mathbf{X}$ & $\mathbf{X}$ & $\mathbf{X}$ & $\mathbf{X}$ \\
\hline Security & $\mathbf{X}$ & $\mathbf{X}$ & & $\mathbf{X}$ & $\mathbf{X}$ \\
\hline Reliability & $\mathbf{X}$ & & $\mathbf{X}$ & $\mathbf{X}$ & \\
\hline Damage to material & $\mathbf{X}$ & & $\mathbf{X}$ & $\mathbf{X}$ & \\
\hline Accuracy & $\mathbf{X}$ & & $\mathbf{X}$ & $\mathbf{X}$ & \\
\hline
\end{tabular}

The weight values of the selected criteria were determined by the direct method for classifying the weights into groups.

The criteria were assigned the weights: 1 . Very important criterion - 9, 2. Medium significant criterion - 6, 3. Less important criterion - 3. The total sum of standard values equals generally 1 .

Table 2. The weight allocation and their transfer to a standard value. Source: authors

\begin{tabular}{|l|c|c|}
\hline \multicolumn{1}{|c|}{ Criteria } & NONSTANDARD VALUE $\mathbf{~} \mathbf{i}$ & STANDARD VALUE $\boldsymbol{\alpha} \mathbf{i}$ \\
\hline Flexibility & 6 & $6 / 60=0,1$ \\
\hline Quick installation & 6 & $6 / 60=0,1$ \\
\hline Economic efficiency & 9 & $9 / 60=0,15$ \\
\hline Capacity utilization & 9 & $9 / 60=0,15$ \\
\hline Security & 9 & $9 / 60=0,15$ \\
\hline Reliability & 9 & $9 / 60=0,15$ \\
\hline Damage to material & 6 & $6 / 60=0,1$ \\
\hline Accuracy & 6 & $6 / 60=0,1$ \\
\hline \multicolumn{1}{|c|}{ Total } & 60 & 1 \\
\hline \multicolumn{1}{|c|}{} & & \\
\hline
\end{tabular}

Partial usefulness is designed by direct access. 1 - does not satisfy the criterion; 2 satisfies the criterion.

Table 3. Classification of individual proposals. Source: authors

\begin{tabular}{|l|c|c|c|c|c|c|c|c|c|c|c|}
\hline \multicolumn{1}{|c|}{ Criteria } & $\boldsymbol{\alpha}_{\mathbf{i}}$ & \multicolumn{1}{c|}{$\mathbf{1}$} & \multicolumn{2}{|c|}{$\mathbf{2}$} & \multicolumn{2}{|c|}{$\mathbf{3}$} & \multicolumn{2}{|c|}{$\mathbf{4}$} & \multicolumn{2}{|c|}{$\mathbf{5}$} \\
\hline Flexibility & 0.1 & 2 & 0.2 & 2 & 0.2 & 2 & 0.1 & 2 & 0.2 & 2 & 0.2 \\
\hline Quick installation & 0.1 & 1 & 0.1 & 2 & 0.2 & 2 & 0.2 & 1 & 0.1 & 1 & 0.1 \\
\hline Economic efficiency & 0.15 & 2 & 0.3 & 2 & 0.3 & 2 & 0.3 & 1 & 0.15 & 1 & 0.15 \\
\hline Capacity utilization & 0.15 & 2 & 0.3 & 2 & 0.3 & 2 & 0.3 & 2 & 0.3 & 2 & 0.3 \\
\hline Security & 0.15 & 2 & 0.3 & 2 & 0.3 & 1 & 0.15 & 2 & 0.3 & 2 & 0.3 \\
\hline Reliability & 0.15 & 2 & 0.3 & 1 & 0.15 & 2 & 0.3 & 2 & 0.3 & 1 & 0.15 \\
\hline Damage to material & 0.1 & 2 & 0.2 & 1 & 0.1 & 2 & 0.2 & 2 & 0.2 & 1 & 0.1 \\
\hline Accuracy & 0.1 & 2 & 0.2 & 1 & 0.1 & 2 & 0.2 & 2 & 0.2 & 1 & 0.1 \\
\hline \multicolumn{1}{|c}{ Total } & 1 & & 1.9 & & 1.65 & & 1.75 & & 1.75 & & 1.4 \\
\hline \multicolumn{1}{|c}{ Ranking } & & & $\mathbf{1 .}$ & & $\mathbf{4 .}$ & & $\mathbf{2 - 3 .}$ & & $\mathbf{2 - 3 .}$ & & $\mathbf{5}$. \\
\hline
\end{tabular}


Table 3 shows that the optimal solution proposal is to add transport lanes in the production hall that was ranked as top-of-the-range. This proposal is effective in order to maintain the current supplying of production workplaces, which would enable overtaking the supply means in the production hall, thus achieving lower times of production requirements fulfilments. This proposal will not have a significant impact on the company's finances, but it brings a high added value in terms of accelerating the supply of production workplaces by supply means. Proposal no. 2 - reducing the number of material unloading has ranked on the fourth place. The advantage is the flexibility that would make it possible to minimize the number of unloading in the production hall in order to improve delivery times by supply means. The disadvantage of this solution is less reliability. The least suitable solution is automated supply means, due to the high initial investment costs.

\section{Conclusion}

The contribution of the article is to point to one of the options to determine the optimal alternative to improve the process of supplying workplaces in the manufacturing company, taking into account the economic efficiency, which is for a company a fundamental condition for implementing the proposed solution. The paper presents the characteristics of the supply means and the proposed solutions for raising efficiency in the supply of production workplaces. The proportional index method evaluates the criteria and the optimal alternative to improve the supply of workplaces.

This work is a part of these projects VEGA 1/0063/16, 1/0403/18, KEGA 018TUKE-4/2016.

This paper is supported by the research project "From horse-drawn railway to intermodal transport" within Visegrad Fund.

\section{References}

1. H. Neradilova, G. Fedorko, Open Engineering 6, 700-710 (2016)

2. M.C. Cooper, D.M. Lambert, J.D. Pagh, Int. J. Logist. Manag. 8, 1-14 (1997)

3. D. Pan, W. Deng, F. Shao, ICLEM 2010, 4424-4430 (American Society of Civil Engineers, Reston, 2010)

4. U. Clausen, M. ten Hompel, M. Klumpp, Efficiency and Logistics (2013)

5. J.T. Mentzer, S. Min, L. Michelle Bobbitt, Int. J. Phys. Distrib. Logist. Manag. 34, 606-627 (2004)

6. N. Brnjac, B. Abramovic, M. Maslaric, Promet-Traffic \& Transp. 22, 303-307 (2010)

7. P. Ficzere, Z. Ultmann, Á. Török, Transport 29, 278-284 (2014)

8. E. Tomková, N. Husáková, J. Strohmandl, Transport Means 2015 - 19th International Conference on Transport Means, 694-697 (2015)

9. B. Chramcov, R. Bucki, S. Marusza, Adv. Intell. Syst. Comput. 210, 423-434 (2013)

10. I. Kubasakova, R. Kampf, O. Stopka, Communications 16, 9-13 (2014)

11. R. Kampf, L. Lizbetinova, K. Tislerova, Open Engineering 7, 26-30 (2017)

12. D. Malindžák, Teória Logistiky (TU Košice, Slovak republic, 2007)

13. A. De Grahl, E. Hartmann, Success Factors in Logistics Outsourcing (2012)

14. H. Neradilova, G. Fedorko, Procedia Engineering 192, 638-643 (2017)

15. CEIT, Available online: https://www.5dimensions.sk/wpcontent/uploads/2015/10/agv-3d-visualization-ceit.jpg (2017) 